\title{
Single versus Dual Antiplatelet Therapy after CABG Surgery
}

\author{
Ryan N. Shely, Gregory S. King*, Marintha R. Short ${ }^{*}$ \\ Department of Pharmacy Services, Saint Joseph Hospital, Lexington, USA. \\ Email: ryan_shely@hotmail.com \\ Received October $12^{\text {th }}, 2013$; revised November $12^{\text {th }}, 2013$; accepted November $20^{\text {th }}, 2013$ \\ Copyright (C) 2013 Ryan N. Shely et al. This is an open access article distributed under the Creative Commons Attribution License, \\ which permits unrestricted use, distribution, and reproduction in any medium, provided the original work is properly cited. In accor- \\ dance of the Creative Commons Attribution License all Copyrights (C) 2013 are reserved for SCIRP and the owner of the intellectual \\ property Ryan N. Shely et al. All Copyright (C) 2013 are guarded by low and by SCIRP as a guardian.
}

\begin{abstract}
Background: The optimal antiplatelet therapy regimen in the early post-operative CABG period is not well researched. Several studies have proven that use of clopidogrel and aspirin prior to surgery increases bleed risks, but very few studies have examined this therapy post-operatively. Due to the limited amount of data surrounding this topic, the goal of this study was to determine if single or dual antiplatelet therapy had better outcomes in those status-post CABG surgery. Methods: This was a retrospective, single center, cohort study performed at Saint Joseph Hospital in Lexington, Kentucky. Data was reviewed through the Society of Thoracic Surgeons (STS) database, pharmacy medication dispensing records, as well as manual chart review. The primary composite endpoint of the study consisted of in-hospital mortality, ischemic or thrombotic events, bleeding events, restenosis rates, and 30-day readmission rates. Results: The number of events with regard to the primary composite endpoint was 32 events with combination therapy and 39 events with aspirin monotherapy $(p=0.39)$. A greater decrease in hemoglobin and hematocrit was seen in the aspirin monotherapy group $(\mathrm{p}=0.02$ and $\mathrm{p}=0.047)$. Patients with prior CVA or TIA were more commonly placed on combination therapy after surgery $(p=0.018)$. There were no differences in outcomes when type of antiplatelet therapy and type of CABG were analyzed. Conclusions: There was no difference seen between single versus dual antiplatelet therapy regarding the primary composite endpoint. There was an increase in bleeding events with aspirin monotherapy as defined by TIMI criteria as well as a statistically significant decrease in Hgb and HCT with aspirin monotherapy. Patients with previous CVA/TIA were more likely to receive combination therapy. The average number of vessels grafted per surgery was lower in the off-pump surgery cohort.
\end{abstract}

Keywords: Coronary Artery Bypass Graft Surgery; CABG, Aspirin; Clopidogrel; Antiplatelet Therapy

\section{Background}

The current standard of care for high risk acute coronary syndrome (STEMI, NSTEMI, and unstable angina) is through the use of percutaneous coronary intervention (PCI). This method of revascularization is much less invasive than coronary artery bypass graft (CABG) surgery and is utilized in most patients who present with these symptoms. However, $10 \%-15 \%$ of this patient population cannot be treated with PCI [1]. The 2011 ACCF/ AHA CABG guidelines state $\mathrm{CABG}$ is the preferred treatment for disease of the left main coronary artery, three vessel disease, and diffuse disease that is not ame-

${ }^{*}$ Corresponding author. nable to treatment with a PCI [2].

Two broad methodologies for CABG surgery exist: one that utilizes the cardiopulmonary bypass machine (on-pump) and another that does not (off-pump). When on-pump surgeries are utilized, the heart is stopped with the use of cardioplegia and blood is circulated through the body via a cardiopulmonary bypass machine. Conversely, off-pump surgeries stabilize the heart with tools but allow normal beats to occur during surgery. Historically, off-pump surgeries were reserved for those with single or double bypass and for those who could not tolerate cardioplegia [3]. However, in present day, these types of surgeries are used interchangeably and depend largely on surgeon preference and the need for cardiac 
repositioning [3]. The recently concluded CORONARY trial followed patients for one year after either on-pump or off-pump surgery and found no difference with respect to the primary composite outcome (rate of death, nonfatal stroke, nonfatal myocardial infarction, or nonfatal new renal failure requiring dialysis at 30 days post-op), the rate of repeat coronary revascularization, quality of life, or neurocognitive function [4].

Patients undergoing off-pump CABG have been found to be prothrombotic, perhaps due to avoidance of the cardiopulmonary bypass machine. Moller and Steinbruchel examined platelet function in on-pump and offpump CABG patients and showed a significant increase in platelet-activating-factor-induced platelet aggregation after off-pump CABG. The cause for this hypercoagulable state is multifarious, but is largely explained by intermittent platelet dysfunction. This dysfunction is ascribed to the coating of platelet surfaces by fibrin degradation products, alpha-degranulation, and loss of surface receptors [5].

Numerous studies have examined post-PCI antiplatelet therapy to prevent stent thrombosis. The standard of care post-PCI is combination therapy with aspirin and a second antiplatelet agent (clopidogrel, prasugrel, ticagrelor, etc.). Despite the establishment of post-PCI antiplatelet therapy, limited data examining post-CABG antiplatelet therapy exists. The $2011 \mathrm{CABG}$ treatment guidelines recommended post-procedural prophylaxis with aspirin initiated within 6 hours post-operatively. For those that are aspirin intolerant, clopidogrel is an acceptable alternative [2]. In contrast to these recommendations, a study performed by Kim and colleagues showed combination therapy was superior to aspirin monotherapy in reducing in-hospital mortality as well as bleeding events in all CABG procedures with no differences seen between on or off-pump cases [1].

Due to the lack of data regarding optimal post-CABG antiplatelet therapy, the goal of this study was to determine if single or dual antiplatelet therapy had better outcomes in those status-post CABG surgery.

\section{Methods}

This was a retrospective, single center, cohort study performed at Saint Joseph Hospital in Lexington, Kentucky. Data was reviewed through the Society of Thoracic Surgeons (STS) database, pharmacy medication dispensing records, as well as manual chart review. The study was granted exempt status by the Western Institutional Review Board prior to data collection. Data was collected for $\mathrm{CABG}$ surgery patients during the time period of July 2011 through December 2011.

The primary composite endpoint of the study consisted of in-hospital mortality, ischemic or thrombotic events, bleeding events, restenosis rates, and 30-day re- admission rates. Secondary endpoints consisted of evaluating each of the components of the primary composite endpoint for separate statistical significance. Ischemic or thrombotic events were defined as cerebral vascular attack (CVA), transient ischemic attack (TIA), or venous thromboembolism (VTE). Bleeding events were defined by the thrombolysis in myocardial infarction (TIMI) bleeding criteria. Restenosis rates were defined by the need for PCI or subsequent CABG surgery to relieve a blockage within 30 days post-operatively.

Patients were eligible for inclusion in the study if they were $>18$ years of age, status-post CABG only surgery (i.e. no concomitant valvular surgery, MAZE procedures, etc.), and received post-operative aspirin with or without clopidogrel within 48 hours after the end of the operation. Patients were excluded if clopidogrel had been used within five days of surgery due to increased bleed risk, use of therapeutic anticoagulation (warfarin, enoxaparin, and heparin), or if there was lack of patient data.

\section{Statistical Analysis}

Data was analyzed using electronic statistical software including Microsoft Excel and MedCalc. Continuous variables were analyzed utilizing the student's t-test. Categorical data was examined using the Fisher's exact and Chi-square tests. A p-value $<0.05$ was considered statistically significant.

\section{Results}

From July 2011 through December 2011, there were 270 eligible patients that received CABG-only procedures. Seventy patients were excluded from the study per the exclusion criteria resulting in a cohort of 200 patients. A total of 100 patients received aspirin monotherapy and a total of 100 patients received dual antiplatelet therapy with aspirin and clopidogrel post-CABG. There were no significant differences in baseline demographic characteristics between the two groups (Table 1).

Regarding the primary composite endpoint, there were a total of 32 events with combination therapy and 39 events with aspirin monotherapy $(\mathrm{p}=0.39)$ (Figure 1). The secondary endpoints showed no statistically significant differences (Table 2). There was an increased incidence in bleeding events with aspirin monotherapy per the TIMI criteria. When bleeding events were evaluated based upon classification, the number of minor bleeds was higher with aspirin monotherapy ( $28 \%$ vs. $18 \%)$, but not statistically significant.

Subsequent data analysis showed a statistically significant difference in hemoglobin and hematocrit changes with greater decreases seen in the aspirin monotherapy group ( $\mathrm{p}=0.02$ and 0.047 ) (Table 3). Patients with prior CVA or TIA were more commonly placed on combina- 


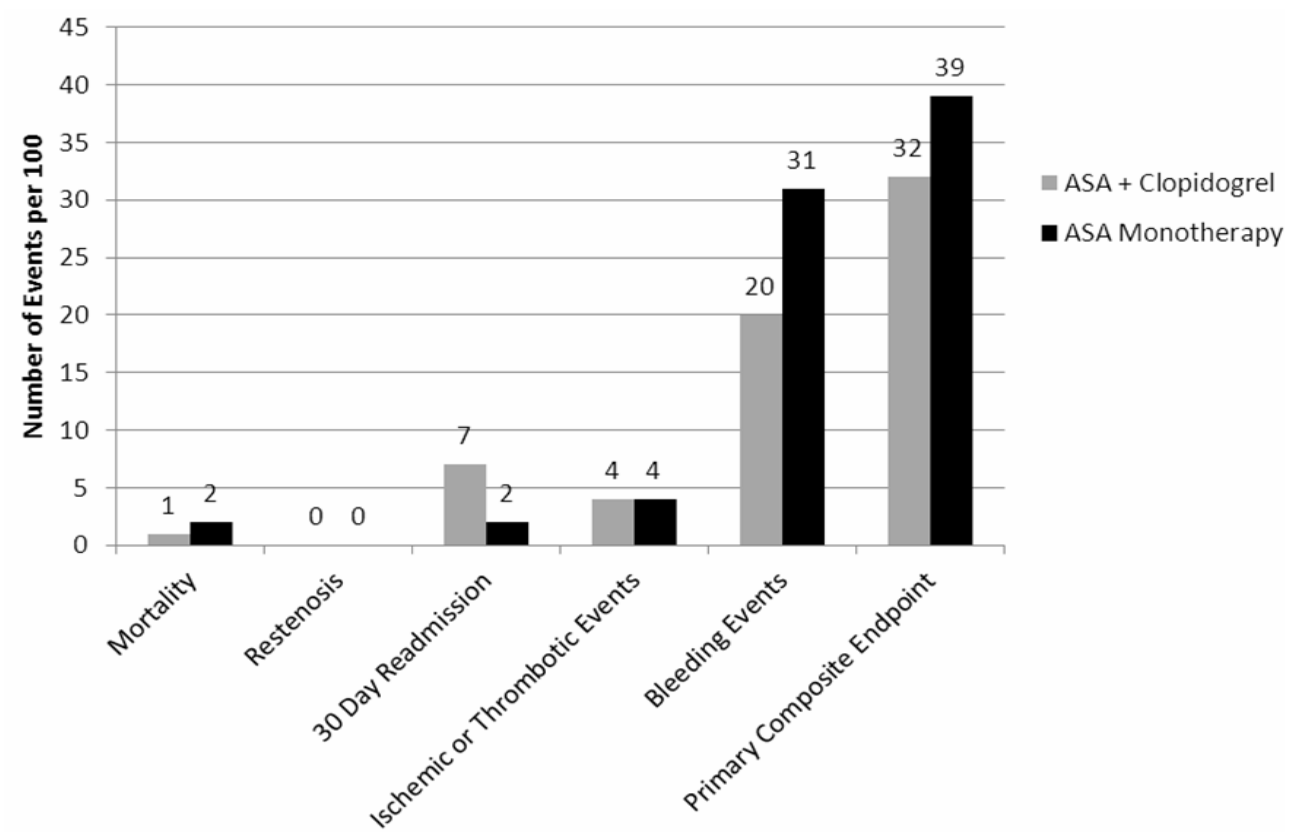

Figure 1. Primary composite endpoint.

Table 1. Demographic data.

\begin{tabular}{cccc}
\hline Category & Dual Therapy $\mathbf{n}=\mathbf{1 0 0}$ & ASA Therapy $\mathbf{n}=\mathbf{1 0 0}$ & p-Value \\
\hline Age \pm SD & $64.47 \pm 9.56$ & $61.99 \pm 11.5$ & 0.069 \\
Male & $71(71 \%)$ & $77(77 \%)$ & 0.33 \\
Height (cm) \pm SD & $173.7 \pm 9.04$ & $173.4 \pm 9.7$ & 0.8 \\
Weight (kg) \pm SD & $92.3 \pm 23.9$ & $89.7 \pm 21.8$ & 0.41 \\
Ethnicity: White (\%) & $96(96 \%)$ & $93(93 \%)$ & 0.35 \\
Prior CHF (\%) & $14(14 \%)$ & $11(11 \%)$ & 0.52 \\
Diabetes (\%) & $48(48 \%)$ & $43(43 \%)$ & 0.47 \\
Previous CABG (\%) & $42(42 \%)$ & $33(33 \%)$ & 0.18 \\
Prior MI (\%) & $47(47 \%)$ & $54(54 \%)$ & 0.32 \\
Platelet Count \pm SD & $215,670 \pm 61,624$ & $211,940 \pm 58,100$ & 0.95 \\
\hline
\end{tabular}

Table 2. Secondary endpoints.

\begin{tabular}{cccc}
\hline Category & Dual Therapy N = 100 & ASA Therapy N = 100 & p-Value \\
\hline In-Hospital Mortality & $1(1 \%)$ & $2(2 \%)$ & 1 \\
Restenosis & $0(0 \%)$ & $0(0 \%)$ & 1 \\
30-Day Readmission & $7(7 \%)$ & $2(2 \%)$ & 0.17 \\
Ischemic or Thrombotic Event & $4(4 \%)$ & $4(4 \%)$ & 1 \\
Bleeding Event & $20(20 \%)$ & $31(31 \%)$ & 0.074 \\
1) Major Bleeding Event & $2(2 \%)$ & $3(3 \%)$ & 1 \\
2) Minor Bleeding Event & $18(18 \%)$ & $28(28 \%)$ & 0.09 \\
\hline
\end{tabular}

tion therapy after surgery $(p=0.018)$. A subgroup analysis of the composite endpoint in on-pump versus offpump surgeries showed no significant difference $(\mathrm{p}=$ 0.93).

When the type of antiplatelet therapy (single versus dual) in on-pump surgeries was analyzed, no difference was seen in composite endpoint $(p=0.26)$. The same was found with off-pump surgeries $(\mathrm{p}=0.39)$. Patients treated with on-pump CABG were statistically more likely to have more vessels grafted than off-pump. In patients that 
Table 3. Secondary analysis.

\begin{tabular}{cccc}
\hline Category & Dual Therapy $\mathbf{( N}=\mathbf{1 0 0})$ & ASA Therapy $\mathbf{( N = 1 0 0 )}$ & p-Value \\
\hline$\beta$-Blocker Use & 85 & 80 & 0.35 \\
ICU Hours \pm SD & $49.5 \pm 47.9$ & $55.06 \pm 36$ & 0.36 \\
LOS (days) \pm SD & $7.14 \pm 3.7$ & $6.88 \pm 3$ & 0.59 \\
$\Delta \mathrm{S}_{\mathrm{Cr}} \pm \mathrm{SD}$ & $0.016 \pm 1.15$ & $0.082 \pm 0.7$ & 0.62 \\
Prior CVA/TIA & 15 & 5 & 0.018 \\
$\Delta \mathrm{Hgb} \pm \mathrm{SD}$ & $-1.63 \pm 1.5$ & $-2.1 \pm 1.3$ & 0.02 \\
$\Delta \mathrm{HCT} \pm \mathrm{SD}$ & $-5.39 \pm 4.1$ & $-6.54 \pm 3.96$ & 0.047 \\
\hline
\end{tabular}

were treated with aspirin monotherapy, a dose of $325 \mathrm{mg}$ was used more often than $81 \mathrm{mg}(62 \%$ vs. $38 \%)$. In patients that received combination therapy, aspirin $81 \mathrm{mg}$ was used more frequently than $325 \mathrm{mg}$ (81\% vs. 19\%).

\section{Discussion}

This study did contain several limitations. Given that this was an observational cohort, there was the possibility for selection bias per prescriber. Those that were deemed to be at high risk for bleeding during surgery may have received aspirin monotherapy to minimize further bleeding risks. Secondly, the TIMI bleeding criteria were used to evaluate bleeding. This may be a less specific indicator of bleeding than chest tube output in the post-operative setting. A previous study by Halkos and colleagues showed higher post-operative chest tube output with early combination therapy versus monotherapy [6]. Patients were followed only while inpatient which did not take into account bleeds or changes in hemoglobin or hematocrit that occurred after discharge. Patients may also have presented to outside facilities after discharge for adverse bleeding events which would not have been trackable.

Another aspect to consider is that the duration of hospitalization in this study was on average 6.5 days. The approximate time for full therapeutic effect of clopidogrel with a $300 \mathrm{mg}$ loading dose is within hours whereas administration of the $75 \mathrm{mg}$ dose takes days [7]. As most patients did not receive a clopidogrel loading doses, the maximal risk of bleeding may only have been monitored for the final days of hospitalization in the combination therapy group. Thus, the majority of data collected assessing bleeding risk may have focused more on the differing strengths of aspirin. As a result, this study may show that bleeding events as measured by the TIMI criteria are higher with aspirin $325 \mathrm{mg}$ versus $81 \mathrm{mg}$. All of these reasons may help explain the differences seen in bleeding risk. However, it is important to note that similar results were seen by Kim and colleagues who utilized a similar study design.

With reference to restenonis and rehospitalization rates, there were no statistically significant differences seen. The dual antiplatelet therapy group did have a higher incidence of readmissions, but these were not directly related to the source of antiplatelet therapy. Reasons for readmission included pneumonia, pleural effusions, sternal wounds, and uncontrolled diabetes. There were no incidences of restenosis during the 30 day post-operative period for either group. In the study performed by Kim and colleagues, total restenotic events were not evaluated. It is possible that our study did not have an adequate sample size to analyze this endpoint.

The subanalysis that compared on-pump surgeries to off-pump surgeries did not show any significant difference in the primary composite endpoint. Again, similar findings were found in the CORONARY trial previously mentioned. It was also concluded that a higher number of vessels were grafted on average in the on-pump surgery group. It is unclear if the differing mean played a significant role in the bleeding findings.

Other limitations to this study include the small sample size is as well as duration of follow-up. The 30 day follow-up period may have been too short to fully detect any benefit of therapy. Future studies that examine combination antiplatelet therapy should consider expanding this window in order to better evaluate if a duration of benefit exists. However, concomitant antiplatelet therapy for an extended period increases the risks for severe bleeds as was seen in the CURE trial [8]. It is unclear at this time what the optimal duration of dual antiplatelet therapy should be.

The study population examined in this trial compared fairly evenly with that of other trials. Specifically compared to Kim and colleagues, the average age and gender in this study were very similar. There were, though, several substantial differences between these populations in race, history of $\mathrm{CHF}$, and history of diabetes. The participants of this study were primarily Caucasian $(97 \%$ versus $\sim 70 \%$ ). Fourteen percent of patients had a history of CHF versus $0.8 \%$, and $48 \%$ of patients had diabetes compared to $39 \%$. Due to the differing and comorbidities, this study may represent new data for a different subset of patients.

\section{Conclusion}

There was no difference seen between single versus dual 
antiplatelet therapy with regard to the primary composite endpoint. A statistically significant decrease in $\mathrm{Hgb}$ and HCT was seen in the aspirin monotherapy group. A higher number of bleeding events was seen in the aspirin monotherapy group as defined by TIMI criteria, a similar finding to what was seen by Kim and colleagues. Patients with previous CVA/TIA were more likely to receive combination therapy. The average number of vessels grafted per surgery was lower in the off-pump surgery cohort.

\section{REFERENCES}

[1] D. H. Kim, C. Daskalakis, et al., "Aspirin and Clopidogrel Use in the Early Postoperative Period Following OnPump and Off-Pump Coronary Artery Bypass Grafting," The Journal of Thoracic and Cardiovascular Surgery, Vol. 138, No. 6, 2009, pp. 1377-1384. http://dx.doi.org/10.1016/j.jtcvs.2009.07.027

[2] L. D. Hillis, P. K. Smith, et al., "2011 ACCF/AHA Guideline for Coronary Artery Bypass Graft Surgery: Executive Summary: A Report of the American College of Cardiology Foundation/American Heart Association Task Force on Practice Guidelines," Circulation, Vol. 124, No. 3, 2011, pp. 1-35.

[3] S. Verma, P. W. M. Fedak, et al., "Off-Pump Coronary Artery Bypass Surgery: Fundamentals for the Clinical
Cardiologist," Circulation, Vol. 109, No. 10, 2004, pp. 1206-1211.

http://dx.doi.org/10.1161/01.CIR.0000120292.65143.F5

[4] A. Lamy, P. J. Devereaux, et al., "Effects of Off-Pump and On-Pump Coronary-Artery Bypass Grafting at 1 Year," NEJM, Vol. 368, No. 13, 2013, pp. 1179-1188. http://dx.doi.org/10.1056/NEJMoa1301228

[5] C. H. Moller and D. A. Steinbruchel, "Platelet Function after Coronary Artery Bypass Grafting: Is There a Procoagulant Activity after Off-Pump Compared with OnPump Surgery?" Scandinavian Cardiovascular Journal, Vol. 37, No. 3, 2003, pp. 149-153. http://dx.doi.org/10.1080/14017430310001456

[6] M. E. Halkos, W. A. Cooper, et al., "Early Administration of Clopidogrel Is Safe after Off-Pump Coronary Artery Bypass Graft Surgery," The Annals of Thoracic Surgery, Vol. 81, 2006, pp. 815-819.

http://dx.doi.org/10.1016/j.athoracsur.2005.08.056

[7] A. Kastrati and A. Schomig, "Are We Making Efficient Use of Clopidogrel," European Heart Journal, Vol. 25, No. 6, 2004, pp. 454-456. http://dx.doi.org/10.1016/j.ehj.2004.02.005

[8] S. Yusuf, F. Zhao, et al., "Effects of Clopidogrel in Addition to Aspirin in Patients with Acute Coronary Syndromes without ST-Segment Elevation," NEJM, Vol. 345, No.7, 2001, pp. 494-502.

http://dx.doi.org/10.1056/NEJMoa010746

\author{
Abbreviations \\ STEMI: ST elevation myocardial infarction \\ NSTEMI: Non-ST elevation myocardial infarction \\ PCI: Pecutaneous Coronary Intervention \\ CABG: Coronary Artery Bypass Graft \\ SCr: Serum Creatinine \\ ICU: Intensive Care Unit \\ MI: Myocardial Infarction \\ CVA: Cerebrovascular Attack \\ TIA: Transient Ischemic Attack \\ TIMI: Thrombolysis in Myocardial Infarction \\ LOS: Length of Stay \\ ASA: Aspirin \\ Hgb: Hemoglobin \\ HCT: Hematocrit
}

\section{TIMI Bleeding Criteria Definition}

\section{Major Bleed}

Intracranial hemorrhage or a $5 \mathrm{~g} / \mathrm{dl}$ decrease in the hemoglobin concentration or a $15 \%$ absolute decrease in the hematocrit.

\section{Minor Bleed}

Observed blood loss: $3 \mathrm{~g} / \mathrm{dl}$ decrease in the hemoglobin concentration or $10 \%$ decrease in the hematocrit.

No observed blood loss: $4 \mathrm{~g} / \mathrm{dl}$ decrease in the hemoglobin concentration or $12 \%$ decrease in the hematocrit.

\section{Minimal Bleed}

Any clinically overt sign of hemorrhage (including imaging) that is associated with a $<3 \mathrm{~g} / \mathrm{dl}$ decrease in the hemoglobin concentration or $<9 \%$ decrease in the hematocrit. 